\title{
Attitudes of clinical staff toward the causes and management of aggression in acute old age psychiatry inpatient units
}

\author{
Terence V McCann ${ }^{1 *+}$, John Baird ${ }^{2 \dagger}$ and Eimear Muir-Cochrane ${ }^{3 \dagger}$
}

\begin{abstract}
Background: In psychiatry, most of the focus on patient aggression has been in adolescent and adult inpatient settings. This behaviour is also common in elderly people with mental illness, but little research has been conducted into this problem in old age psychiatry settings. The attitudes of clinical staff toward aggression may affect the way they manage this behaviour. The purpose of this study was to examine the attitudes of clinical staff toward the causes and management of aggression in acute old age psychiatry inpatient settings.
\end{abstract}

Methods: A convenience sample of clinical staff were recruited from three locked acute old age psychiatry inpatient units in Melbourne, Australia. They completed the Management of Aggression and Violence Scale, which assessed the causes and managment of aggression in psychiatric settings.

Results: Eighty-five staff completed the questionnaire, comprising registered nurses (61.1\%, $n=52$ ), enrolled nurses $(27.1 \%, n=23)$ and medical and allied health staff $(11.8 \%, n=10)$. A range of causative factors contributed to aggression. The respondents had a tendency to disagree that factors directly related to the patient contributed to this behaviour. They agreed patients were aggressive because of the environment they were in, other people contributed to them becoming aggressive, and patients from certain cultural groups were prone to these behaviours. However, there were mixed views about whether patient aggression could be prevented, and this type of behaviour took place because staff did not listen to patients. There was agreement medication was a valuable approach for the management of aggression, negotiation could be used more effectively in such challenging behaviour, and seclusion and physical restraint were sometimes used more than necessary. However, there was disagreement about whether the practice of secluding patients should be discontinued.

Conclusions: Aggression in acute old age psychiatry inpatient units occurs occasionally and is problematic. A range of causative factors contribute to the onset of this behaviour. Attitudes toward the management of aggression are complex and somewhat contradictory and can affect the way staff manage this behaviour; therefore, wide-ranging initiatives are needed to prevent and deal with this type of challenging behaviour.

Keywords: Aggression, Attitudes, Elderly, Old age psychiatry, Nurses, Restraint, Seclusion, Survey

\footnotetext{
* Correspondence: terence.mccann@vu.edu.au

${ }^{\dagger}$ Equal contributors

'Discipline of Mental Health Nursing and Aged Care, College of Health and

Biomedicine, Victoria University, PO Box 1428, Melbourne 8001, Victoria,

Australia

Full list of author information is available at the end of the article
}

\section{Biomed Central}

(c) 2014 McCann et al.; licensee BioMed Central Ltd. This is an Open Access article distributed under the terms of the Creative Commons Attribution License (http://creativecommons.org/licenses/by/2.0), which permits unrestricted use, distribution, and reproduction in any medium, provided the original work is properly credited. 


\section{Background}

Aggression ('any form of behaviour that is intended to injure someone physically or psychologically' [1], p.6) occurs commonly in elderly people, aged 65 years and older, with mental illness and is mainly associated with dementia [2]; for instance, 15-43\% of community referrals to old age psychiatry services are because of aggression [3]. Staff working in inpatient units for elderly people with organic mental illness are more likely to be to be assaulted than those working in other inpatient settings [4]. When aggression occurs in inpatient settings it is more likely to be directed at nurses than other patients $[2,5]$, or at other clinical and non-clinical staff [4], and rarely culminates in severe injury $[2,6,7]$. Little research has been undertaken to examine the attitudes of staff toward patient aggression in acute old age psychiatry inpatient units. It is important to examine these attitudes because they may affect the way staff attempt to prevent and manage this behaviour. This paper adds to the literature on aggression by presenting the findings of a survey of clinical staff attitudes toward this behaviour in inpatient units for elderly people with mental health problems in Australia.

\section{Causes of aggression}

The causes of aggression in elderly patients with mental health problems are complex and interrelated. Aggression may be attributable to psychosocial-environmental factors or the complex interaction of patients, staff and inpatient unit culture influences [8]. It may be due to the inability of elderly patients with dementia to communicate their needs effectively [9], poor staff-to-patient interactions or the environment of care $[9,10]$, overcrowding, lack of privacy, lack of activities, weak clinical leadership [11], being denied something [7], and restricting patients' freedom [12].

There is also some debate that aggression may be due to the elderly person having a pre-morbid personality trait of aggression. Various reviews and studies have concluded, however, that an inconclusive relationship exists between premorbid personality and this behaviour $[13,14]$. For instance, a systematic review conducted by Osborne, Simpson and Stokes [14] concluded that 72\% of studies detected significant relationships between premorbid personality - particularly pre-morbid neuroticism - and challenging behaviour such as aggression; however, several studies found no relationship. In addition, there is a direct association between patients with Alzheimer's disease who have a recent history of aggression [15] and childhood behavioural problems.

There are also contrasting reports about a relationship between particular illnesses and aggression. On the one hand, there are claims that aggression is associated with the person's illness [9]. In particular, positive relationships have been reported between certain symptomatology, such as paranoid delusional thinking [5,16], impulsivity [17], dementia symptoms [2], depressive symptoms [13], and aggression. In contrast, James et al. [18] found no diagnosis was positively associated with aggression in an acute psychiatric ward.

\section{Restraint and seclusion}

When confronted by aggression, clinical staff may use a range of person-centred (e.g., good staff-to-patient communication, distraction, de-escalation) and containment (e.g., restraint, seclusion, medication) measures to manage this behaviour. Two containment approaches - restraint and seclusion - are examined because there is debate about their use in the literature. Restraint (the restriction of a person's freedom of movement by physical, mechanical, chemical, and/or emotional means [19]) and seclusion (the sole confinement of a person in a room where the doors and windows are locked [20]) may be used to prevent and manage aggression [21] but they can have adverse effects on elderly patients in old age psychiatry in particular. Both practises are contrary to prominent international recommendations [22], government reports, mental health service policies, service user organisations and scholarly literature, which advocate that as strategies to deal with disturbed behaviour, restraint and seclusion should be used as little as possible or eliminated.

International variation is evident in containment practises, and this is influenced by a range of factors such as concerns about efficacy and patient safety, and broader cultural values [23]. For instance, in the United Kingdom mechanical restraint is not used and is regarded by many nurses to be reprehensible [23]; in Finland, overall, seclusion is used more frequently than mechanical restraint, but there are regional variations in these practises [24]. In Australia, while there are no national data on the use of restraint and seclusion, the reduction and potential elimination of restraint and seclusion practises and adverse events have been a recommendation of the recent National Mental Health Commission [25], and have been identified as one of four key national priority areas for increasing safety and reducing harm in mental health care [26]. In acute old age psychiatry inpatient settings in particular, restraint use has come under increased examination as a consequence of evidence it may be deleterious [26], ineffective, and unnecessary [27]. The overuse of restraint and seclusion is regarded as an early sign of a mental health system under pressure [25].

Research into the use of restraint in elderly people has been conducted in general hospitals [28] and nursing home settings $[9,10,29]$. However, there has been little investigation of the use of these containment practices 
with elderly persons in old age psychiatry inpatient settings, and the rate and type of restraint use can vary, even within neighbouring units [30]. Furthermore, Cochrane reviews of restraint and seclusion [31] and containment practises [32] have concluded that nonpharmacological approaches to restraint and seclusion are not supported by evidence from controlled studies.

\section{Attitudes toward aggression}

The attitudes of clinical staff toward aggression can influence the way they respond to this behaviour [33,34]. Positive attitudes may influence the adoption of personcentred approaches whereas negative attitudes may contribute to the use of containment measures. Studies undertaken in various settings have provided contradictory findings about the attitudes of staff toward patient aggression. A United Kingdom survey in a high secure hospital, by Pulsford et al. [35], highlighted that staff held a range of attitudes about the causes and management of patient aggression. However, a United Kingdom survey of the attitudes of staff toward aggressive older people with dementia in residential care, by Pulsford et al. [10], noted that this behaviour was attributable to interpersonal problems resulting from unfavourable situational events such as an adverse environment of care and poor interaction with others in the setting. Furthermore, the staff response to aggression was mainly to adopt a person-centred approach rather than measures such as restraint, seclusion and medication. In contrast, a survey of staff attitudes toward patient aggression in dementia facilities in Japan, by Nakahira et al. [36], reported a significant relationship between staff with negative attitudes toward aggressive patients and the use of physical and chemical restraint. Similarly, Duxbury \& Whittington [37], in a United Kingdom survey of staff attitudes toward aggression in an acute psychiatric inpatient setting, highlighted that respondents who perceived internal influences on patients contributed to this behaviour, such as the nature of their illness, were more likely to use containment than person-centred methods to manage this behaviour. The implication of possessing negative attitudes toward aggression and adoption of containment measures is these approaches can culminate in adverse health outcomes for elderly patients in particular. For example, a survey and data set analysis of all nursing homes $(\mathrm{N}=740)$ in Pennsylvania in the United States found a significant association between physical restraint use and subsequent deterioration in patients' cognitive and activities of daily living performance and increased walking dependence $[9,10,29]$.

Little research has been carried out into the attitudes of clinical staff toward the causes and management of patient aggression in psychiatry in general $[33,34]$ and in acute old age psychiatry in particular [2]. In light of the relationship between attitudes toward aggression and the adoption of measures to deal with this behaviour, and the implications for the patient's wellbeing, the purpose of this study was to examine the attitudes of clinical staff toward the causes and management of aggression in acute old age care psychiatry inpatient settings.

\section{Method}

Study design

A survey design was used, incorporating a structured questionnaire.

\section{Sample \& setting}

A convenience sample of clinical staff was recruited from three locked acute old age psychiatry inpatient units and their associated community outreach teams, in Melbourne, Australia. The units are managed by the same public mental health service, but each is located in separate geographical locations. The units offer mainly single-room accommodation with en suite toilets, and all have common recreational and dining facilities and gardens. Patients aged 65 years and over are admitted directly to the units from the community or residential care for short-term management of an acute phase of mental illness, until they recover enough to be treated in a community-based setting, and are different, therefore, from nursing homes that cater specifically for people with dementia. Medical, nursing and allied health staff provide the care, and the staffing ratios are broadly similar across the units.

The inclusion criterion for staff was clinical staff employees (Unit manager, Registered/enrolled nurses, psychologists, social workers, occupational therapists, psychiatrists) working on day shifts in the respective units. The exclusion criterion was staff working at night and at weekends. Staff received written invitations to participate, and the project was explained to them either at staff meetings or individually. They were given the choice to complete the questionnaire and hand it back to the researcher, or to return it by mail. Almost all chose the former option.

\section{Instrument}

The Management of Aggression and Violence Scale (MAVAS) [37-39] was used to assess attitudes toward the causes of, and ways to manage, aggression. It originally contained 27 items, on a four-point Likert scale, ranging from 1 (strongly agree) to 4 (strongly disagree), with the cut-off for agreeing set at 2.5 (A 5-point version of the scale is also available). A low score indicates agreement with a statement. The Scale has undergone psychometric evaluation and has been shown to contain a strong four-factor structure: internal, external and situational/interactional influences on aggression [38]. (i) Internal $(n=5)$ : Aggression is due mainly to factors within 
the aggressive person (e.g., mental illness or personality); (ii) External ( $n=3)$ : Aggression is caused mainly by influences in the person's physical or social environment (e.g., physical layout of ward, or manner in which the ward is managed by staff); (iii) Situational/interactional $(n=5)$ : Aggression is attributable to factors in the immediate environment, such as the way staff interact with patients; (iv) Approaches to the management of aggression $(n=14)$ : (e. g., use of medications, restraint and seclusion). The most recent version of the instrument, which was used in the present study, contains 30 items; the three additional items focus on cultural/gender issues.

The internal consistency of the instrument has been established in several studies [38-40]. The Cronbach's alpha in the present study was 0.8. Ideally, Cronbach's alpha should be above 0.7 [41].

\section{Ethics}

Ethical approval to carry out the study was obtained from Melbourne Health Mental Health Research and Ethics Committee. Researchers, who were not employees of the mental health service, undertook recruitment. Return of questionnaires was interpreted as consent.

\section{Statistical analysis}

Data analyses were undertaken using the $\mathrm{R}$ environment for statistical computing and graphics [42]. Frequencies, percentages, means and standard deviations were used to analyse the socio-demographic characteristics of respondents. Means and standard deviations were used to assess responses to the causes and management of aggression.

\section{Results}

Socio-demographic characteristics of participants

Approximately 90 clinical staff were invited to take part in the study, and of these, 85 completed the questionnaire, equivalent to $78 \%$ of the total number of staff in the three units. Unit 3 had the highest level of participation, followed by units 1 and 2 respectively. Almost two-thirds of respondents were female. The mean age of respondents was 43 years, ranging from 24 to 62 years. About half were born in Australia, just over $40 \%$ in Asian countries, and just under $10 \%$ in western European countries. Most respondents $(88 \%)$ were registered and enrolled nurses, while the remainder were medical and allied health staff (Table 1).

\section{Causes and management of patient aggression}

The results are presented in two main sections: (i) causes of aggression, and (ii) management of this behaviour.

\section{Causes of aggression Internal factors}

The overall mean score for internal causes of aggression was $2.6(\mathrm{SD}=0.4)$, suggesting respondents had a tendency
Table 1 Socio-demographic characteristics of participants $(\mathrm{N}=\mathbf{8 5})$

\begin{tabular}{|c|c|c|c|c|}
\hline & & & $\mathbf{n}$ & $\%$ \\
\hline \multirow[t]{2}{*}{ Gender } & \multicolumn{2}{|l|}{ Male } & 29 & 34.1 \\
\hline & \multicolumn{2}{|l|}{ Female } & 56 & 65.9 \\
\hline \multirow[t]{3}{*}{ Occupation } & \multicolumn{2}{|c|}{ Registered nurse } & 52 & 61.1 \\
\hline & \multicolumn{2}{|c|}{ Enrolled nurse } & 23 & 27.1 \\
\hline & \multicolumn{2}{|c|}{ Psychiatrist/Allied health } & 10 & 11.8 \\
\hline \multirow[t]{3}{*}{ Unit } & \multicolumn{2}{|l|}{1} & 26 & 30.6 \\
\hline & \multicolumn{2}{|l|}{2} & 22 & 25.9 \\
\hline & \multicolumn{2}{|l|}{3} & 37 & 43.5 \\
\hline \multicolumn{5}{|c|}{ Country/region of birth } \\
\hline \multicolumn{3}{|l|}{ Australia } & 40 & 47.6 \\
\hline \multicolumn{3}{|l|}{ Asia } & 36 & 42.9 \\
\hline \multicolumn{3}{|l|}{ Western Europe } & 8 & 9.5 \\
\hline \multirow[t]{2}{*}{ Age (years) $(n=81)$} & $M$ & SD & Min & Max \\
\hline & 43.1 & 11.3 & 24 & 62 \\
\hline
\end{tabular}

to disagree that factors directly attributable to the patient contributed to this type of behaviour. Specifically, they perceived aggression was associated with mental illness, and particular types of patients were prone to aggression. They also indicated aggressive behaviour was preventable, but tended to disagree that such behaviour resolved of its own accord if the patient was left alone (Table 2).

\section{External factors}

The overall mean score for external causes was 2.0 (SD = 0.4 ), indicating respondents tended to agree that environmental factors in the units were influential in causing aggression. Restrictive care environments, such as locked wards, were perceived as contributing to aggression. Likewise, respondents were in agreement that if the physical environment was better patients would be less likely to be aggressive (Table 2).

\section{Situational/interactional factors}

The overall mean score for situational/interactional causes of aggression was $2.1(\mathrm{SD}=0.5)$, suggesting respondents were in agreement that factors in the immediate situation, including the way staff communicated with patients, contributed to this type of behaviour. In particular, the respondents were in agreement that patient aggression occurred because of the influence of others. Patients were also more likely to become aggressive because of poor patient-to-staff communication. However, there was agreement-to-disagreement that this form of challenging behaviour was attributable to staff failing to listen to patients (Table 2). 
Table 2 Means and standard deviation (SD) of beliefs about the cause of aggression

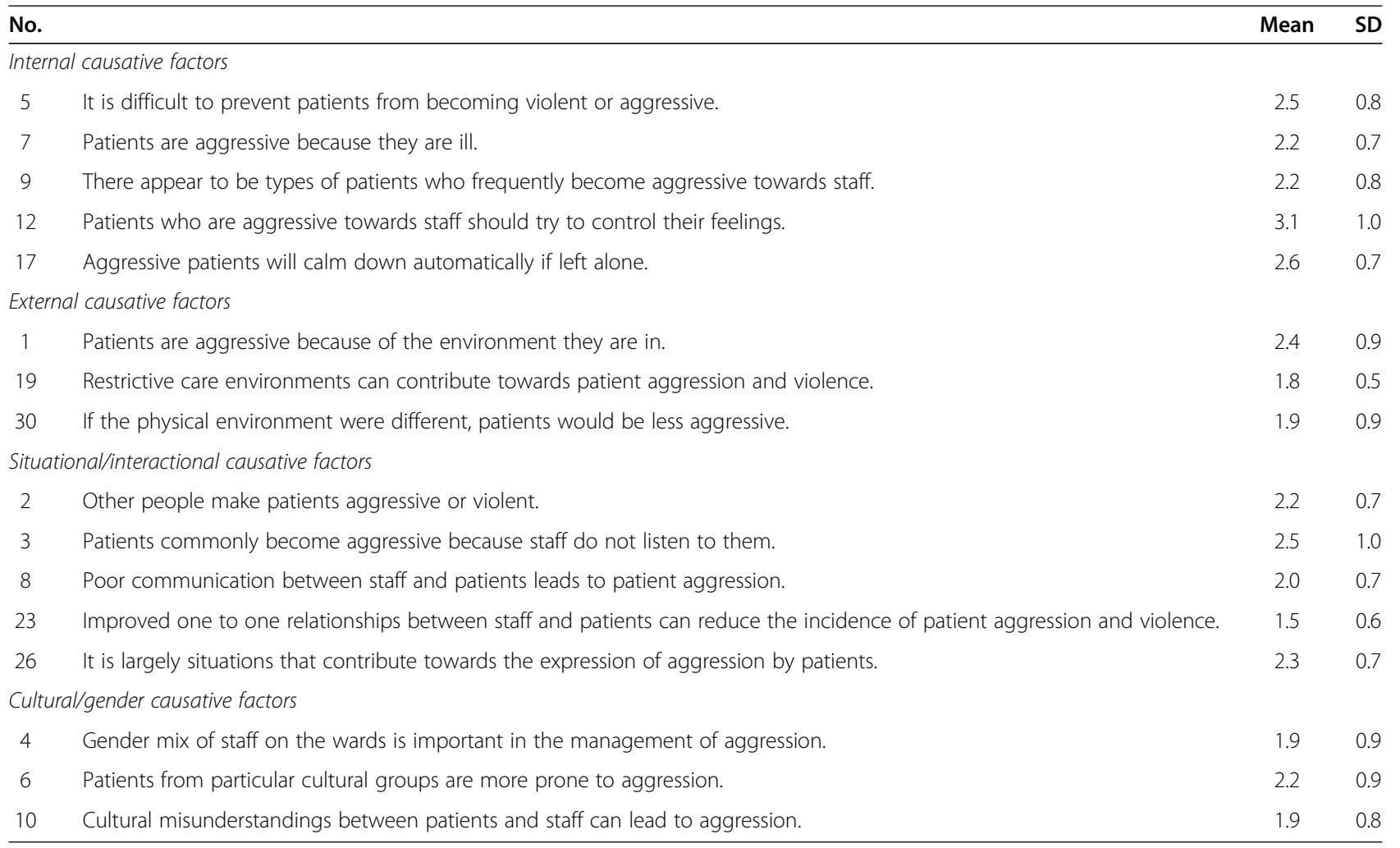

Rating scale: 1 = strongly agree, 2 = agree, $3=$ disagree, $4=$ strongly disagree.

\section{Cultural/gender factors}

The overall mean score of $2.0(\mathrm{SD}=0.7)$ suggested respondents were in general agreement with the statements that cultural and gender influences contributed to the initiation of aggression in units. In particular, there was agreement patients' cultural background and cultural miscommunications between patients and staff contributed to the onset of aggression. Likewise, there was agreement gender mix of staff was an important consideration in dealing with aggression (Table 2).

\section{Management of aggression}

The overall mean score for the management of this type of behaviour was $2.3(\mathrm{SD}=0.3)$, indicating respondents had a tendency to agree with the statements about how to respond to these challenging situations (Table 3). Generally, there was agreement patient aggression could be dealt with more effectively in the units. In particular, there was agreement medication was useful for treating aggression and it should be used more frequently with patients who displayed this behaviour. However, they also responded that in some circumstances medication contributed to instances of aggression.

The findings indicated agreement-to-disagreement that seclusion was one of the most effective ways for dealing with this form of behaviour, restraint and seclusion were sometimes used more often than necessary, and patients were usually restrained for their own safety. Moreover, there was disagreement with the statement that the practice of seclusion should be discontinued.

There was general agreement person-centred alternatives to containment and sedation, such as negotiation and de-escalation, could be used more effectively to prevent and manage aggression. However, there was agreement-to-disagreement about whether or not staff should always intervene in situations when patients were aggressive.

\section{Discussion}

The findings of our study provide a valuable insight into the attitudes of clinical staff respondents toward the contentious issue of the causes and management of aggression in acute old age psychiatry inpatient settings. In relation to the causes, overall, the respondents tended to disagree that internal or direct patient related factors were contributing influences. However, they perceived aggression was related to mental illness, and some patients were more susceptible to aggression than others. These findings suggest that while the respondents were less likely to attribute blame to patients for aggression, they perceived those with certain conditions were more susceptible to these forms of behaviours. This view is 
Table 3 Means and standard deviation (SD) of beliefs about the management of aggression

\begin{tabular}{|c|c|c|c|}
\hline \multicolumn{2}{|c|}{ No. } & \multirow[t]{2}{*}{ Mean } & \multirow[t]{2}{*}{ SD } \\
\hline Manc & nt: General & & \\
\hline 11 & Different approaches are used on this ward to manage patient aggression and violence. & 1.7 & 0.8 \\
\hline 24 & Patient aggression could be handled more effectively on this ward. & 2.1 & 0.8 \\
\hline \multicolumn{4}{|c|}{ Management: Use of medication } \\
\hline 16 & Medication is a valuable approach for treating aggressive and violent behaviour. & 2.3 & 0.9 \\
\hline 25 & Prescribed medication can in some instances lead to patient aggression and violence. & 2.2 & 0.6 \\
\hline 28 & Prescribed medication should be used more frequently to help patients who are aggressive and violent. & 2.4 & 0.9 \\
\hline \multicolumn{4}{|c|}{ Management: Use of seclusion } \\
\hline 13 & When a patient is violent, seclusion is one of the most effective approaches to use. & 2.7 & 0.9 \\
\hline 15 & The practice of secluding violent patients should be discontinued. & 3.0 & 0.8 \\
\hline 27 & Seclusion is sometimes used more than necessary. & 2.5 & 0.9 \\
\hline \multicolumn{4}{|c|}{ Management: Restraint } \\
\hline 14 & Patients who are violent are often restrained for their own safety. & 2.5 & 0.9 \\
\hline 21 & Physical restraint is sometimes used more than necessary. & 2.4 & 0.9 \\
\hline \multicolumn{4}{|c|}{ Management: Non-physical methods } \\
\hline 18 & Negotiation could be used more effectively when managing aggression and violence. & 1.9 & 0.7 \\
\hline 20 & Expressions of aggression do not always require staff intervention. & 2.5 & 0.8 \\
\hline 22 & Alternatives to the use of containment and sedation to manage patient violence could be used more frequently. & 2.1 & 0.7 \\
\hline 29 & The use of de-escalation is successful in preventing violence. & 1.7 & 0.6 \\
\hline
\end{tabular}

consistent with some literature suggesting patients with persecutory delusions [5,16] and impulsivity [17] are particularly prone to aggression; the risk is greater during the acute phase of psychotic illness; and thought disorder, impairment of neuropsychological functioning, disorganized behaviour and substance misuse, are lesser contributing factors $[2,13,43-45]$.

The respondents in the current study also indicated socio-demographic influences; in particular, cultural consideration had contrasting effects on the onset of aggression. They felt patients from some cultural backgrounds were more susceptible to aggression than others, and cultural misinterpretations between staff and patients contributed to this behaviour. There are contrasting findings in the limited psychiatric literature examining the influence of cultural background on aggression. Depp [46] found black patients are overrepresented in the striking role whereas white patients are more likely to be in the non-striking role. However, James et al. [18] reported there were no statistical differences between the cultural backgrounds of violent and non-violent patients. Therefore, in the present study cultural misinterpretations may be attributed to poor staff-to-patient interactions $[9,10]$, which can lead to frustration and this, in turn, increases the likelihood of aggression [1]. They may also be due to cultural misunderstandings wherein some illness related behaviours, such as aggression and loud speech, are perceived as abnormal by clinicians but may be perfectly acceptable within a particular patient's culture [47].

The respondents agreed that gender mix of staff on wards was helpful in the prevention of aggression. There are contrasting findings in the literature, however, about the influence of staff gender on patient aggression. Daffern et al. [48], in a 6-month review of episodes of patient aggression in a forensic psychiatric hospital, found no statistically significant relationship between the gender ratio of staff and aggression. However, a review of ecological factors influencing inpatient psychiatric unit violence, by Hamrin et al. [8], presented conflicting findings about staff gender and aggression; with some studies concluding male staff were at greater risk of being recipients of violence, whereas other studies reported females staff were the most common recipients of violence.

The respondents in the present study tended to agree that external and situtational/interactional (or situational and contextual) influences contributed to the onset of aggression, including the use of restrictive environments such as locked wards. They also perceived if the physical environment was improved patients would be less prone to aggression. These findings can be explained by external and situational/interactional influences, such as restrictive environments, which increase the likelihood of frustration and the possibility 
of aggression [1]. The findings also accord with other studies that report the physical characteristics of the ward environment, such as irritating noise, lack of privacy, restriction in liberty $[12,43,44,49]$, and lack of activities [11], contribute to aggression.

There was agreement in the current study that poor staff-to-patient communication contributed to the onset of aggression. Whittington and Wykes [50], in a United Kingdom study of inpatient aggression, also reported the influence of aversive stimulation by staff, such as physical contact, frustration, demands patients participate in activities, and critical comments. It can also be extrapolated that poor staff-to-patient communication leads to frustration $[1,9,10]$, which, in turn, may culminate in patient aggression.

Regarding the management of patient aggression, the respondents were in agreement this behaviour could be managed more successfully, and a person-centred approach could be used more often as well as judicious use of medication. These findings can be explained, whereby external stimuli, such as person-centred and careful medication-use techniques [10,11], could have a moderating influence in reducing the likelihood of aggression [1].

The findings of the present study also indicated restraint and seclusion were used more than necessary in the management of aggression, and there was disagreement about whether the practice of seclusion should be stopped. The preference by staff to retain the option to use seclusion has also been reported in United Kingdom studies by Duxbury and Whittington [37] and Foster et al. [51]. Possible explanations for the somewhat contradictory findings in the current study are personcentred and pharmacological approaches are perceived as insufficient to deal with all instances of aggression. Another explanation is staff support for these containment practises is affected adversely by workplace situational and contextual and broader cultural influences and is resistant to change. To illustrate, support for such practises may be lower in countries where these approaches are uncommon but higher in countries where they are adopted more often, such as Australia [21,23,52]. Furthermore, the current study's findings highlight a dichotomy between government $[25,26]$, service user $[19,52]$ and carer groups [19] that advocate the abolition of seclusion and the views of coalface face clinicians confronted with this type of challenging behaviour.

\section{Limitations and strengths}

While a representative sample of $78 \%$ of clinical staff was obtained, the data may not accurately reflect the views of all such staff because those working at weekends and at night were not included. This could be addressed in a future study by recruiting respondents throughout the 24-hour, 7-day spectrum. Next, as the sample was derived from three inpatient units within a single mental health service, this limits the ability to infer from this sample to the general population of clinical staff working in other acute old age psychiatry inpatient units. Recruiting respondents from a wider range of mental health services could rectify this in a subsequent study. Finally, the sample only included the views of clinical staff; current and former inpatients were considered for inclusion but were not deemed well enough by clinical staff to provide consent.

\section{Conclusions}

Our survey provides a valuable insight into the contentious issue of attitudes toward the causes and management of aggression, and contributes to the limited body of research about this issue in old age psychiatry inpatient settings. Consideration of attitudes is important because there is a direct relationship between attitudes and the types of measures that are used to manage this challenging behaviour. It can be inferred from the findings of this study that a broad approach to prevention and management of aggression - one that addresses socio-demographic and interpersonal as well as situational and contextual influences - needs to be adopted. They findings also show clinical staff possess contrasting attitudes about the most effective and acceptable ways to prevent and manage this challenging behaviour; in particular, there were mixed views about whether the practice of seclusion should cease.

Therefore, measures taken to address prevention and management should also focus on these contrasting attitudes. Overall, strategies to prevent and manage aggression in acute old age psychiatry inpatient settings have implications for the overall quality of care; in particular, patient charactristics, inpatient environment, the way staff interact with patients, cultural sensitivity and gender mix of staff, and consideration of appropriate ways to manage aggression such as the adoption of personcentred alternatives to restraint and seclusion.

\section{Competing interests}

The authors declare that they have no competing interests.

\section{Authors' contributions}

TMCC had a major role in the design of the study, undertook some data collection, carried out the data analysis, and had a major role in writing the paper. JB had a major role in the design of the study, and had a key role in writing the paper. EMC had a major role in the design of the study, and had a major role in writing the paper. All authors have approved the final draft.

\section{Acknowledgements}

Our sincere appreciation to Flora McCann for her major contribution to data collection. Our thanks to Dr Neil Diamond, Victoria University, Melbourne, for his statistical advice. 


\section{Funding}

The authors declare receipt of the following financial support for the study: The study was funded by a grant from the Helen Macpherson Smith Trust, Melbourne, Australia.

\section{Author details}

'Discipline of Mental Health Nursing and Aged Care, College of Health and Biomedicine, Victoria University, PO Box 1428, Melbourne 8001, Victoria, Australia. ${ }^{2}$ NorthWestern Mental Health Old Aged Persons' Mental Health Program, Harvester Building, 4C Devonshire Road, Sunshine 3020, Victoria, Australia. ${ }^{3}$ School of Nursing and Midwifery, Flinders University, GPO Box 2100, Adelaide, South Australia 5001, Australia.

Received: 24 January 2013 Accepted: 12 March 2014

Published: 19 March 2014

\section{References}

1. Berkowitz L: Aggression: It's causes, consequences, and control. New York: McGraw-Hill; 1993.

2. Chaplin R, McGeorge M, Hinchcliffe G, Shinkwin L: Aggression on psychiatric inpatient units for older adults and adults of working age. Int J Geriatr Psychiatry 2008, 23(8):874-876.

3. O'Callaghan CE, Richman AV: Violence in older people with mental illness. Adv Psychiatr Treat 2010, 16:339-348.

4. Royal College of Psychiatrists' Centre for Quality Improvement: The Healthcare Commission National Audit of Violence 2006-7. Final report - Older people's services. London: Royal College of Psychiatrists; 2008. http:/www. rcpsych.ac.uk/PDF/OP\%20Nat\%20Report\%20final\%20for\%20Leads.pdf.

5. Cornaggia CM, Beghi M, Pavone F, Barale F: Aggression in psychiatry wards: a systematic review. Psychiatry Res 2011, 189(1):10-20.

6. Astrom S, Karlsson S, Sandvide A, Bucht G, Eisemann M, Norberg A, Saveman BI: Staff's experience of and the management of violent incidents in elderly care. Scand J Caring Sci 2004, 18(4):410-416.

7. Almvik R, Rasmussen K, Woods P: Challenging behaviour in the elderlymonitoring violent incidents. Int J Geriatr Psychiatry 2006, 21(4):368-374

8. Hamrin V, lennaco J, Olsen D: A review of ecological factors affecting inpatient psychiatric unit violence: implications for relational and unit cultural improvements. Issues Ment Health Nurs 2009, 30(4):214-226.

9. Duxbury J, Pulsford D, Hadi M, Sykes S: Staff and relatives' perspectives on the aggressive behaviour of older people with dementia in residential care: A qualitative study. J Psychiatr Ment Health Nurs 2013, 20(9):792-800.

10. Pulsford D, Duxbury JA, Hadi M: A survey of staff attitudes and responses to people with dementia who are aggressive in residential care settings. J Psychiatr Ment Health Nurs 2011, 18(2):97-104.

11. National Institute for Health and Clinical Excellence: Dementia: The NICE-SCIE guidelines on supporting people with dementia and their carers in health and social care (National Clinical Practice Clinical Guideline Number 42). In The British Psychological Society \& Gaskell; 2011. http://www.nice.org.uk/ nicemedia/live/10998/30320/30320.pdf.

12. Papadopoulos C, Ross J, Stewart D, Dack C, James K, Bowers L: The antecedents of violence and aggression within psychiatric in-patient settings. Acta Psychiatr Scand 2012, 125(6):425-439.

13. Pulsford D, Duxbury J: Aggressive behaviour by people with dementia in residential care settings: a review. J Psychiatr Ment Health Nurs 2006, 13(5):611-618

14. Osborne H, Simpson J, Stokes G: The relationship between pre-morbid personality and challenging behaviour in people with dementia: A systematic review. Aging Ment Health 2010, 14(5):503-515.

15. O'Leary $D$, Jyringi $D$, Sedler M: Childhood conduct problems, stages of Alzheimer's disease, and physical aggression against caregivers. Int $J$ Geriatr Psychiatry 2005, 20(5):401-405.

16. Lewis $C F$, Fields $C$, Rainey E: A study of geriatric forensic evaluees: who are the violent elderly? J Am Acad Psychiatry Law 2006, 34(3):324-332.

17. Ferguson CJ, Averill PM, Rhoades H, Rocha D, Gruber NP, Gummattira P. Social isolation, impulsivity and depression as predictors of aggression in a psychiatric inpatient population. Psychiatr Quart 2005, 76(2):123-137

18. James DV, Fineberg NA, Shah AK, Priest RG: An increase in violence on an acute psychiatric ward. A study of associated factors. Br J Psychiatry 1990, 156:846-852.
19. National Mental Health Consumer Carer Forum: Statement on seclusion and restraint in mental health services: April 2009; 2009. http://www.nmhccf.org. au/documents/Seclusion\%20\&\%20Restraint.pdf.

20. Parliament of Victoria: Mental Health Act 1986 (Reprint No. 6). In Act No 59/1986. Melbourne: Victoria Government Publication; 1986.

21. Bowers L, Alexander J, Simpson A, Ryan C, Carr-Walker P: Cultures of psychiatry and the professional socialization process: the case of containment methods for disturbed patients. Nurse Educ Today 2004, 24:435-442.

22. Sailas $E$, Wahlbeck K: Restraint and seclusion in psychiatric inpatient wards. Curr Opin Psychiatry 2005, 18:555-559.

23. Bowers $L$, van der Werf B, Vokkolainen A, Muir-Cochrane E, Allan T, Alexander J: International variation in containment measures for disturbed psychiatric inpatients: a comparative questionnaire survey. Int J Nurs Stud 2007, 44(3):357-364

24. Korkeila JA, Tuohimäki C, Kaltiala-Heino R, Lehtinen V, Joukamaa M Predicting use of coercive measures in Finland. Nord J Psychiatry 2002, 56(5):339-345.

25. National Mental Health Commission: A Contributing Life, the 2012 National Report Card on Mental Health and Suicide Prevention. Sydney: National Health and Medical Research Council; 2012. http://www.mentalhealth commission.gov.au/media/39270/NMHC ReportCard Enhanced.pdf.

26. National Mental Health Working Group: National safety priorities in mental health: A national plan for reducing harm. Canberra: Department of Health and Ageing, Commonwealth of Australia; 2005. http://www.health.gov.au/ internet/publications/publishing.nsf/Content/mental-pubs-n-safety-toc.

27. Weintraub $D$, Spurlock M: Change in the rate of restraint use and falls on a psychogeriatric inpatient unit: impact of the health care financing Administration's new restraint and seclusion standards for hospitals. J Geriatr Psychiatry Neurol 2002, 15(2):91-94.

28. Strumpf NE, Evans LK: Physical restraint of the hospitalized elderly: perceptions of patients and nurses. Nurs Res 1988, 37(3):132-137.

29. Engberg J, Castle N, McCaffrey D: Physical restraint initiation in nursing homes and subsequent resident health. Gerontologist 2008, 48(4):442-452.

30. O'Connor D, Horgan L, Cheung A, Fisher D, George K, Stafrace S: An audit of physical restraint and seclusion in five psychogeriatric admission wards in Victoria, Australia. Int J Geriatr Psychiatry 2004, 19(8):797-799.

31. Sailas EES, Fenton M: Seclusion and restraint for people with serious mental illnesses (Review). Cochrane Database Syst Rev. Cochrane Collaboration 2012(6). 2012. doi:10.1002/14651858.CD001163 [http://www. mrw.interscience.wiley.com/cochrane/clsysrev/articles/CD001163/frame.html]

32. Muralidharan S, Fenton M: Containment strategies for people with serious mental illness. In Cochrane Database Syst Rev. Cochrane Collaboration; 2012. Issue 2. doi:10.1002/14651858.CD002084.pub2 http://www.mrw. interscience.wiley.com/cochrane/clsysrev/articles/CD002084/frame.html.

33. Jansen GJ, Dassen TWN, Johannes GM, Burgerhof GM, Middel B: Psychiatric nurses' attitudes towards inpatient aggression: preliminary report of the development of Attitude Towards Aggression Scale (ATAS). Aggress Behav 2006, 32:44-53.

34. Jansen GJ, Middel B, Dassen TW, Reijneveld MS: Cross-cultural differences in psychiatric nurses' attitudes to inpatient aggression. Arch Psychiatr Nurs 2006, 20(2):82-93.

35. Pulsford D, Crumpton A, Baker A, Wilkins T, Wright K, Duxbury J: Aggression in a high secure hospital: staff and patient attitudes. J Psychiatr Ment Health Nurs 2013, 20:296-304.

36. Nakahira M, Moyle W, Creedy D, Hitomi H: Attitudes toward dementiarelated aggression among staff in Japanese aged care settings. J Clin Nurs 2009, 18(6):807-816.

37. Duxbury J, Whittington R: Causes and management of patient aggression and violence: staff and patient perspectives. J Adv Nurs 2005, 50(5):469-478

38. Duxbury J: Testing a new tool: the management of aggression and violence scale (MAVAS). Nurse Res 2003, 10(4):39-52.

39. Duxbury J, Hahn S, Needham I, Pulsford D: The Management of Aggression and Violence Attitude Scale (MAVAS): a cross-national comparative study. $J$ Adv Nurs 2008, 62(5):596-606.

40. Hahn S, Needham I, Abderhalden C, Duxbury JA, Halfens RJ: The effect of a training course on mental health nurses' attitudes on the reasons of patient aggression and its management. J Psychiatr Ment Health Nurs 2006, 13(2):197-204

41. DeVellis RF: Scale development: theory and applications. 2nd edition. Thousand Oaks, California: Sage; 2003. 
42. R Development Core Team: A Language and Environment for Statistical Computing. Vienna, Austria: R Foundation for Statistical Computing; 2011

43. Daffern M, Howells K: Psychiatric inpatient aggression: a review of structural and functional asessment approaches. Aggress Violent Behav 2002, 7:477-497.

44. Nijman HL: A model of aggression in psychiatric hospitals. Acta Psychiatr Scand Suppl 2002, 106(s412):142-143.

45. Steinert T, Wolfle M, Gebhardt RP: Measurement of violence during in-patient treatment and association with psychopathology. Acta Psychiatr Scand 2000, 102(2):107-112.

46. Depp FC: Violent behavior patterns on psychiatric wards. Aggress Behav 1976, 2:295-306.

47. Bhugra D: Severe mental illness across cultures. Acta Psychiatr Scand 2006, 113(Suppl. 429):17-23.

48. Daffern M, Mayer M, Martin T: Staff gender ratio and aggression in a forensic psychiatric hospital. Int J Ment Health Nurs 2006, 15(2):93-99.

49. Wong SE, Slama KM, Liberman RP: Behavioral analysis and therapy for aggressive psychiatric and developmentally disabled patients. In Clinical treatment of the violent person. Edited by Roth LH. New York: Guilford Press; 1987:20-53.

50. Whittington $R$, Wykes $T$ : Aversive stimulation by staff and violence by psychiatric patients. Br J Clin Psychol 1996, 35(1):11-20.

51. Foster C, Bowers L, Nijman H: Aggressive behaviour on acute psychiatric wards: prevalence, severity and management. J Adv Nurs 2007, 58(2):140-149.

52. Veltkamp E, Nijman H, Stolker JJ, Frigge K, Dries P, Bowers L: Patients' preferences for seclusion or forced medication in acute psychiatric emergency in the Netherlands. Psychiatr Serv 2008, 59(2):209-211.

doi:10.1186/1471-244X-14-80

Cite this article as: McCann et al: Attitudes of clinical staff toward the causes and management of aggression in acute old age psychiatry inpatient units. BMC Psychiatry 2014 14:80.

\section{Submit your next manuscript to BioMed Central and take full advantage of:}

- Convenient online submission

- Thorough peer review

- No space constraints or color figure charges

- Immediate publication on acceptance

- Inclusion in PubMed, CAS, Scopus and Google Scholar

- Research which is freely available for redistribution 